\title{
Determination of Plasma Retinol by High Performance Liquid Chromatography, and its Significance in Digestive Diseases
}

\author{
Kose Segawa, Saburo Nakazawa, Yoshihisa Tsukamoto, \\ Hatuhiro Yamaguchi, Hidemi Goto and Yasumitsu Kurita
}

\begin{abstract}
Plasma retinol was determined by reverse-phase high-performance liquid chromatography in healthy subjects and patients with digestive diseases. The plasma retinol in the healthy control was independent of age $(80.5 \pm 17.16 \mu \mathrm{g} / \mathrm{dl})$. On the other hand, the amount of retinol in patient groups was lower than in the healthy control group. It was $30.96 \pm 7.16 \mu \mathrm{g} / \mathrm{dl}$ in patients with liver cancer, $38.02 \pm 11.63 \mu \mathrm{g} / \mathrm{dl}$ in those with chronic pancreatitis and pancreatic cancer, $45.36 \pm 12.63 \mu \mathrm{g} / \mathrm{dl}$ in a patient group with inflammatory bowel disease, $66.25 \pm 7.27 \mu \mathrm{g} / \mathrm{dl}$ in the patients of early gastric cancer, and $66.44 \pm 10.10$ $\mu \mathrm{g} / \mathrm{dl}$ in the group with biliary stone. These results and many biological aspects of retinol in human made it clear that, the special attention should be paid to the fluctuation of plasma retinol amount. This was considered to play an important role in causing complications associated with digestive diseases.
\end{abstract}

Key Words: Plasma retinol, Reptic ulcer, Inflammatory bowel disease, Liver cancer, Pancreatitis, Pancreatic cancer.

Retinol (vitamin A) is a polyisoprenoid compound containing a cyclohexenyl ring. It is essential in higher animals for support of growth and health. This vitamin plays important roles especially in vision, reproduction, mucus secretion and maintenance of differentiated epithelia ${ }^{1}$. The role of retinol in diseases of digestive organ has also gained attention, but the assay procedures for plasma retinol by former method $^{2)}$ were troublesome, and the results were sometimes incorrect. Recently, high-performance liquid chromatography (HPLC) was adopted successfully to separate many fat-soluble vitamins including retinol, the procedure became relatively easy, and the results of the assay became more reliable ${ }^{3)}$. The results of plasma retinol determined by HPLC in health and digestive diseases are reported in order to elucidate the differences in the retinol metabolism in healthy subjects and in patients with digestive diseases. The role of plasma retinol in the pathophysiology of digestive diseases is also discussed.

\section{MATERIALS AND METHODS}

\section{Subjects}

The subjects $(n=95)$ for this study consisted of healthy controls $(n=61,47.46 \pm 8.22$ mean \pm SD years old), patients with pancreatic disease $(n=9$, $53.78 \pm 9.41$ years old, 6 with calcified pancreatitis and 3 with pancreatic cancer), inflammatory bowel disease involving small intestine and colon in active stage $(n=4,45.00 \pm 20.26$ years old), early gastric cancer $(n=9,62.00 \pm 10.45$ years old), liver cancer $(n=5,58.00 \pm 7.69$ years old), and bile duct stone $(n=7,56.00 \pm 16.35$ years old) (Table). All but the subjects were out-

From The Second Department of Internal Medicine, Nagoya University School of Medicine.

(Director: Prof. Tatsuo Satake)

Received for publication July 12, 1985.

Reprint request to: Kose Segawa, MD, The Second Department of Internal Medicine,

Nagoya University School of Medicine, 65 Tsuruma-cho, Showa-ku, Nagoya 466, Japan. 
Table The Subjects for Study

\begin{tabular}{lrcr}
\cline { 2 - 4 } Control & 61 & $34: 27$ & $47.46 \pm 8.22$ \\
Pancreatic ca, P-tits & 9 & $7: 2$ & $53.78 \pm 9.41$ \\
Inflammatory Bowel Dis. & 4 & $3: 1$ & $45.00 \pm 20.26$ \\
Early Gastric Cancer & 9 & $7: 2$ & $62.00 \pm 10.45$ \\
Liver Cancer & 5 & $4: 1$ & $58.00 \pm 7.69$ \\
Bile Duct Stone & 7 & $3: 4$ & $56.00 \pm 16.35$ \\
\hline Total Number & 95 & $58: 37$ &
\end{tabular}

and in-patients of our hospital. The healthy controls were examinee who underwent precise examinations including blood chemistry, barium meal, chest X-ray, ECG, urinary and fecal examination, and were proven to be healthy in a certain health care institution.

\section{Methods}

Sample: The blood samples were obtained in the morning after an overnight fast and were submitted to the determination of retinol and retinol binding protein by the following procedures.

Assay of Retinol in Plasma: $200 \mu \mathrm{l}$ of a subject's plasma was diluted with $1 \mathrm{ml}$ of twice distilled water, then $1 \mathrm{ml}$ of ethanol was added. Subsequently, $5 \mathrm{ml}$ of hexane was added to the mixture and stirred vigorously for 5 minutes. The mixture was centrifuged at $5,000 \mathrm{rpm}$ at $4^{\circ} \mathrm{C}$ for 5 minutes. The upper layer $(4 \mathrm{ml})$ was taken out in small test tube, and the extract was subsequently dried up under the stream of $\mathrm{N}_{2}$ gas at $30^{\circ} \mathrm{C}$. The residue was taken up in $100 \mu \mathrm{l}$ of isopreterenol, and then an aliquot $(25 \mu \mathrm{l})$ of sample was injected into the HPLC system.

The apparatus for retinol determination system used in this study was obtained from Shimadzu Seisakusho, Co., Ltd. (Kyoto, Japan). The highperformance liquid chromatography (Model LC$5 \mathrm{~A})$ was equipped with fluorescence photometer (RF-530). The excitation wavelength was set at $340 \mathrm{~nm}$ and the emission was set at $460 \mathrm{~nm}$. A reverse-phase column, Zorbax-ODS (4.6 × 150 $\mathrm{mm}$ ), was used. Figure 1 shows the chromatogram of retinol prepared from human plasma (1-b) and standard sample (1-a). In each chromatogram, retinol appeared at 7 minutes after injecting the

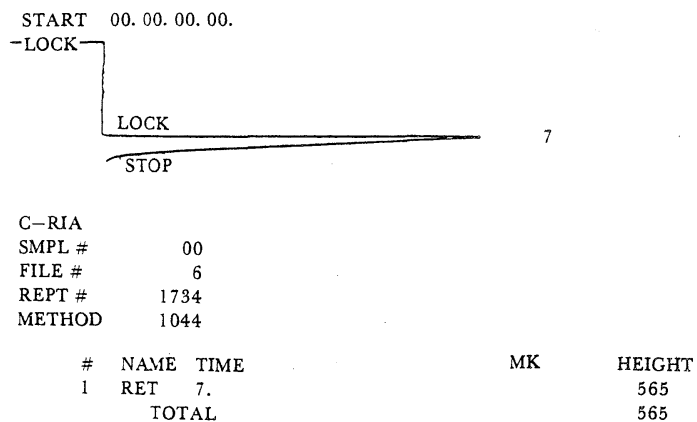

Fig. 1-a

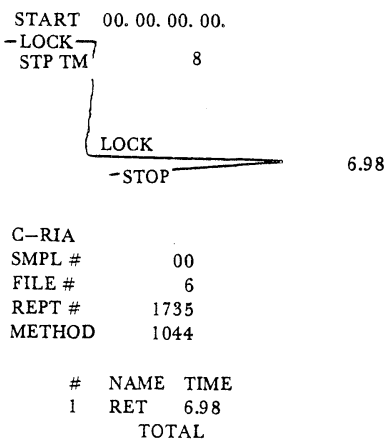

Fig. 1-b

Fig. 1. The chromatogram of retinol by high performance liquid chromatography. Mobile phase flow rate: $0.5 \mathrm{ml} / \mathrm{min}$; pressure: $80 \mathrm{~kg} / \mathrm{cm}^{2}$; wavelength: excitation $=340 \mathrm{~nm}$; emission $=460 \mathrm{~nm}$; range: 16 ; column temperature: $30^{\circ} \mathrm{C}$; chart speed: $0.25 \mathrm{~cm} / \mathrm{min}$; Fig. 1-a: Standard of retinol (50 ng). Fig. 1-b: Sample prepared from plasma of normal control group $(25 \mu \mathrm{l})$.

sample,and no impurities associated with retinol were found.

Assay of Retinol Binding Protein (RBP): RBP was assayed by a radial immuno-diffusion technique using a commercial kit, L-C Partigen (pur- 
chased from Behringwerke AG, Marburg, West Germany) following the method of Mancini ${ }^{4)}$. The plasma from human blood was diluted with two volumes of saline solution $(0.9 \% \mathrm{NaCl})$, and $25 \mu \mathrm{l}$ of the diluted sample was subject to determination according to the kit instruction.

\section{Statistics}

Statistical analysis was carried out using modified t-test, after the differences among groups were confirmed to be significant by variance analysis ${ }^{5)}$. The difference between means was considered significant when $\mathrm{p}<0.05$. The relationship between the amount of plasma retinol and RBP was tested by linear regression analysis. The relationship was considered significant when $\mathrm{p}<0.05$.

\section{RESULTS}

When the plasma retinol was determined by HPLC as described in "MATERIALS AND METHODS", the amount of retinol in blood plasma was $80.51 \pm 3.44$ (mean $\pm \mathrm{SEM}$ ) $\mu \mathrm{g} / \mathrm{dl}$ in the group of healthy control. The details of the plasma retinol value in various age groups were slightly different. The values were $93.59 \pm 8.96$ $\mu \mathrm{g} / \mathrm{dl}$ in the group under 39 years old $(\mathrm{n}=11)$, $78.82 \pm 5.68 \mu \mathrm{g} / \mathrm{dl}$ in the fourth decade age group $(\mathrm{n}=24)$, and $76.53 \pm 4.42 \mu \mathrm{g} / \mathrm{dl}$ in the group older than 50 years old $(n=26)$ (Figure 2). However, there were no statistically significant differences

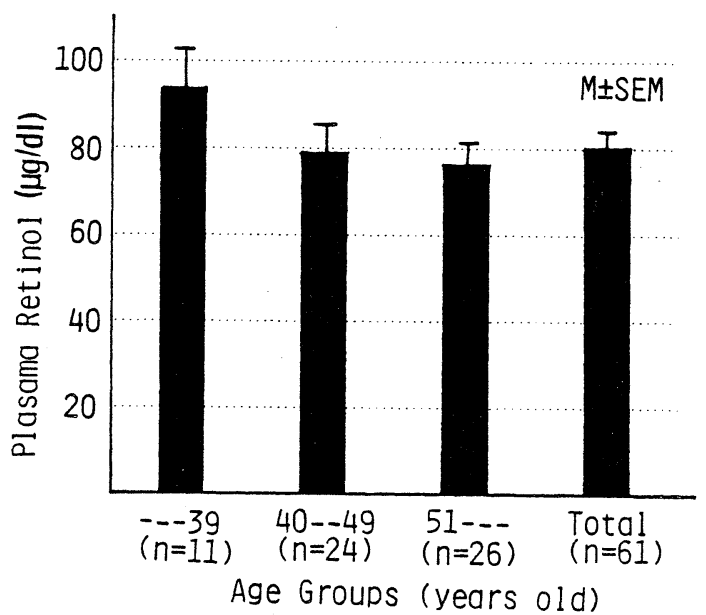

Fig. 2. The plasma level of retinol in the various age groups. There were no statistically significant differences among them. in the quantity of plasma retinol in each age group.

The amount of plasma retinol in ill patients was lower than that in healthy controls. The value was $30.96 \pm 7.16 \mu \mathrm{g} / \mathrm{dl}$ in patients with liver cancer, $38.02 \pm 11.63 \mu \mathrm{g} / \mathrm{dl}$ in chronic pancreatitis and pancreatic cancer, $45.36 \pm 12.63 \mu \mathrm{g} / \mathrm{dl}$ in inflammatory bowel disease, $66.25 \pm 7.27 \mu \mathrm{g} / \mathrm{dl}$ in early gastric cancer, and $66.44 \pm 10.10 \mu \mathrm{g} / \mathrm{dl}$

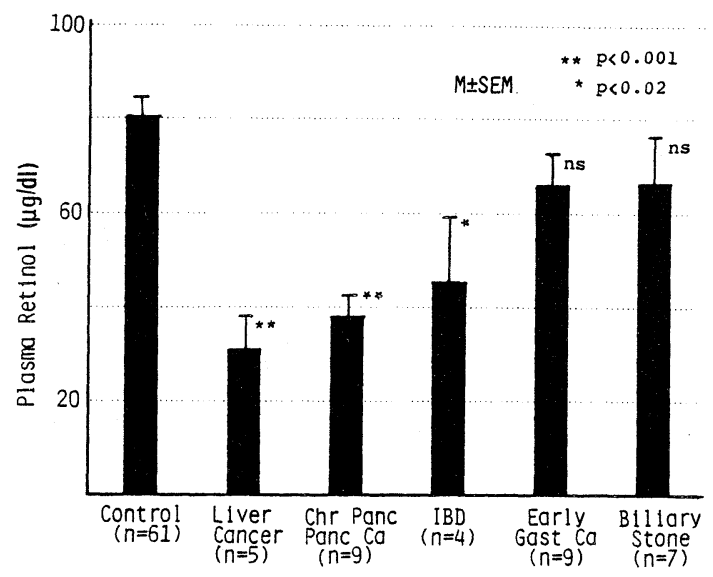

Fig. 3. The plasma level of retinol in various digestive diseases. There was a statistically significant difference between control group and liver cancer group ( $\mathrm{p}<0.001)$; control group and chronic pancreatitis-pancreatic cancer group ( $p<0.001)$; control group and inflammatory bowel disease (IBD) group $(\mathrm{p}<0.02)$.

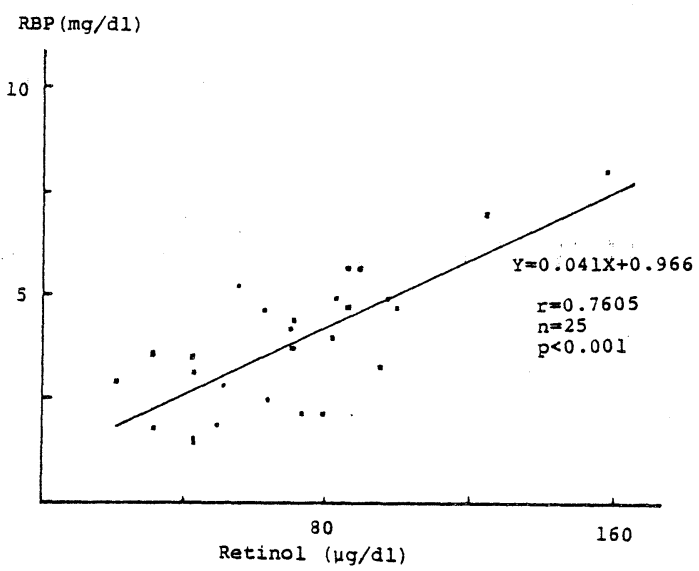

Fig. 4. A linear correlationship $(r=0.7605)$ existed between plasma retinol level and retinol binding protein (RBP). The relationship was statistically significant $(\mathrm{p}<0.001)$. 
in biliary stone group (Figure 3). All the plasma retinol in the patient groups showed low values which reflected statistically significant differences between the healthy controls and liver cancer ( $p$ $<0.001$ ), pancreatic disease, or inflammatory bowel disease group ( $p<0.001),(\mathrm{p}<0.02)$ respectively.

There was a statistically significant correlationship between the plasma retinol level and $\mathrm{RBP}(\mathrm{r}=0.7605, \mathrm{p}<0.01)$ as shown in Figure 4.

\section{DISCUSSION}

Formerly, retinol in serum or plasma has been determined with the fluorescence method based on molecular fluorescence properties of the retinol structure ${ }^{2)}$. Although the fluorescence method could detect retinol in plasma, the presence of fluorescent components other than retinol raised problems of nonspecificity with serum samples. Thus, the fluorescence impurities contained in plasma sample were also additive to the fluorescence intensity of retinol. As a result, the fluorescence intensity was larger than the exact value of the material. To overcome this problem, a chromatographic step was introduced before fluorometry ${ }^{3)}$, as was performed in the present study.

In developed countries, vitamin A deficiency is unlikely to occur for dietary reasons alone ${ }^{6}$. In our study, the plasma retinol level which was determined by HPLC was $80.50 \pm 17.16 \mu \mathrm{g} / \mathrm{dl}$ in the healthy controls. When the normal control group was divided into three groups (younger, under 39 years old; middle age, from 40 to 49 , and older group over 50), the average amount of plasma retinol in each group increased with the age. However, there were no statistically significant differences among the age groups. While the plasma retinol level was apparently lower in the liver cancer, inflammatory bowel disease, and pancreatic disease than in the normal control group, the decrease of retinol could be explained as follows.

In animal products, dietary vitamin $\mathrm{A}$, or retinol has the long chain fatty acid ester of retinol. In vegetables, it exists as a provitamin in the form of $\beta$-carotenes ${ }^{7)}$. The retinol is absorbed from the small intestine with fat, enters the lymph stream and travels to the liver with chylomicron ${ }^{8)}$. The damage to the small intestine or pancreas sometimes decreases the absorption ability. This can lead to a low blood vitamin A level, as was shown in the present study. Goodman injected intravenously chylomicrons containing newly absorbed vitamin A into normal intact rats, and observed the tissue distribution of radioactivity for a several days ${ }^{9}$. They found approximately two-thirds of the recovered radioactivity in the liver, and confirmed the major role of the liver in the metabolism of newly absorbed vitamins. Thus, the liver is the main storage place in the body for vitamin A. Liver diseases of various types may therefore be expected to interfere in one one way or another with the metabolism and physiological activity of vitamin A. Retinyl ester, which is a form of storage in the liver, is hydrolyzed to free retinol and fatty acid; then the retinol is bound with $\mathrm{RBP}$, which is synthesized in the hepatocyte. The retinol- and RBP-protein complex, called holoretinol-binding protein, secretes out of the liver, then enters the circulation, and delivers retinol to the target tissues ${ }^{10}$. When the disease has disordered the metabolism of liver cell, secretion of retinol- and RBP-complex is comparatively disturbed. Consequently, the concentration of retinol and RBP in plasma of patients is lower in the presence of liver disease than that in healthy control.

Xerosis in various forms, and defective dark adaptation, which was reported to be associated with liver disease ${ }^{11,12)}$ or Crohn's disease ${ }^{6,13)}$ might result from reduced vitamin A level.

Peptic ulcer disease is highly complicated with liver cirrhosis or other hepatic failure ${ }^{14,15)}$. Vitamin A significantly inhibited the development of gastric mucosal lesions produced by $0.6 \mathrm{M} \mathrm{HCl}$, but had no inhibitory effect on gastric acid secretion in 4 hour pylorus-ligated $\mathrm{rat}^{16)}$. The decreased level of plasma retinol associated with depressed liver function may play one of the important roles in the etiology of peptic ulcer disease in hepatic failure, such as liver cirrhosis or hepatoma.

The results of the present paper indicates that patients with digestive disease, such as liver disease and malabsorption syndrome including 
pancreatic and intestinal disease, should be managed with consideration for the plasma or serum retinol level, which plays various important roles in the maintenance of human biologic activity. For further development of this study, more detailed investigations should be performed on the mechanism of reduction or loss of the plasma level of retinol.

\section{REFERENCES}

1) Martin DW: Fat soluble vitamins, chap 11. Harper's Review of Biochemistry (Martin DW, Mayes PA and Rodwell VW, eds), 18th edition, Lange Medical Publications, Los Altos, p 114, 1981.

2) Kahan J: A method for the fluorometric determination of vitamin A. Scand J Clin Lab Invest 18: 679, 1966.

3) De Ruyter MGM: Determination of serum retinol (vitamin A) by high-speed liquid chromatography. Clin Chem 22: 1593, 1976.

4) Mancini G, Carbonara AO, Heremans JF: Immunochemical quantitation of antigens by single radial immunodiffusion. Immunochemistry 2: 235, 1965.

5) Wallenstein S, Zucker CL, Fleiss JL: Some statistical method useful in circulation research. Circulation Research 47: 1, 1980.

6) Thompson $A B R$ and Dietschy: Intestinal lipid absorption: Major extracellular and intracellular events, chap 46. Physiology of Gastrointestinal Tract (Johnson LR, ed), Raven Press, New York, p 1147,
1981.

7) Mandel HG: Fat soluble vitamins, chap 75. Basis of Therapeutics (Goodman LS and Gilman A, eds), 5th edition, Macmillan Publishing Co, New York, p 1570, 1975.

8) Main ANH, Mills PR, Russel RI, et al: Vitamin A deficiency in Crohn's disease. Gut 24: 1169, 1983.

9) Goodman DS, Hsuang HS, Shiratori T, et al: Tissue distribution and metabolism of newly absorbed vitamin A in the rat. J Lipid Research 6: 390, 1965.

10) Smith FR and Goodman DS: The effects of diseases of the liver, thyroid, and kidneys on the transport of vitamin A in human plasma. J Clin Invest 50: $2426,1971$.

11) Harris $\mathrm{AD}$ and Moore $\mathrm{T}$ : Vitamin $\mathrm{A}$ in infective hepatitis. Brit Med J 1: 553, 1947.

12) Russel RM, Morrison SA, Smith FR, et al: Vitamin A reversal of abnormal dark adaptation in cirrhosis study of effects on the plasma retinol transport system. Ann Int Med 88: 622, 1978.

13) Vahlquist A, Sjolund K, Norden A, et al: Plasma vitamin A transport and visual dark adaptation testing in diseases of the intestine and liver. Scand J Clin Lab Invest 38: 301, 1978.

14) Swisher WP, Baker LA, Bennetr HD: Peptic ulcer in Laennec's cirrhosis. Amer J Dig Dis 22: 291, 1955.

15) Tabaqchali S, Dawson AM: Peptic ulcer and gastric secretion in patients with liver disease. Gut 5: 417, 1964.

16) Javor T, Bata M, Lovasz L, et al: Gastric cytoprotective effects of vitamin A and other carotenoids. Int J Tiss Reac 5: 289, 1983. 